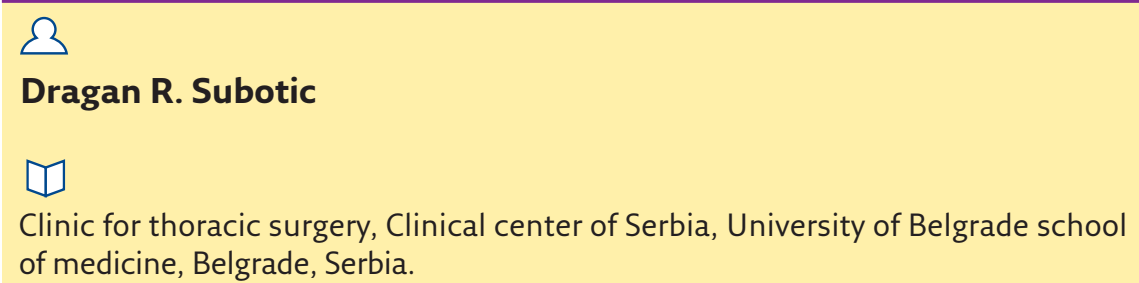

Clinic for thoracic surgery, Clinical center of Serbia, University of Belgrade school of medicine, Belgrade, Serbia.

\title{
Key Questions in Thoracic Surgery
}

\author{
Authors: Narain Moorjani, Nicola Viola and William S. Walker; \\ TFM Publishing; 1040 pages; ISBN: 9781903378861
}

\section{Book review}

This 1000-page textbook encompasses much more than the title suggests. In fact, the title "Key questions in thoracic surgery and pulmonology" would be more fitting. The specific format of the book, with precise questions and evidence-based, but equally clear answers covering all relevant fields of pulmonology and thoracic surgery, makes this 40-chapter book a "must read" not only for residents, but also for senior pulmonologists and thoracic surgeons.

Wherever you open this book, you will see questions such as "What is ventilation-perfusion ratio?"; "What is the alveolar-arterial pressure gradient?"; "What are the principals of management of stage III lung cancer?"; and "What are the indications for surgery for cystis fibrosis?". These questions look quite ordinary and are frequently asked, but the answers are so clear that they simply "push" the reader to keep on reading, more and more, chapter after chapter. Given that at least a third of the book is taken up by top quality figures, schematics and images, the effective size of the book is not as excessive as it may seem at first sight.

There are four main pillars of this book. The first, basics in clinical anatomy, respiratory physiology and pathophysiology, is so well tailored that it meets most of the needs of modern clinicians. The second, diagnostic procedures, is an up-to-date overview of all noninvasive and invasive procedures in pulmonology and thoracic surgery. It is completely education-oriented with clear examples and explanations of high-resolution computed tomography (CT), positron emission tomography, ultrasound, endoscopic procedures and surgical diagnostic tools. The section on preoperative assessment is particularly well done. Diagnostic procedures are also dispersed and incorporated within the third pillar, clinical considerations of particular diseases, with guidelines for diagnostic and therapeutic approaches. This integrated approach makes this book unique, deleting the boundaries between surgery and pulmonology. The fourth pillar, which represents individual surgical procedures within this integrated text, looks like it was designed to keep all respiratory physicians informed about surgery as one of the treatment options, rather than to present surgery itself as an isolated, technology- and skill-based specialty. Of course, the surgical procedures are described in detail, as the book is designed primarily for surgeons. Furthermore, an overview
Cite as: Subotic DR. Book review: Key Questions in Thoracic Surgery. Breathe 2016; 12: 175-176.

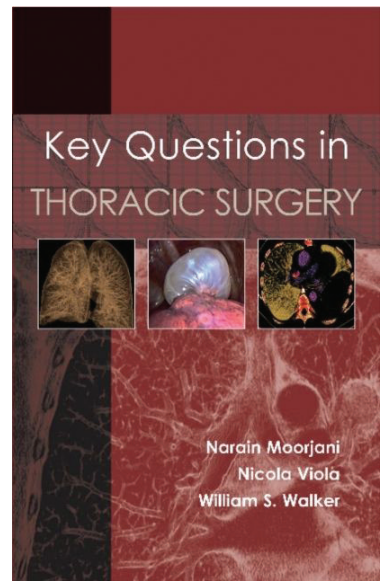


of standard and novel (video-assisted) surgical tools to be used in diagnostics is presented so as to identify which tools should be used for which disease. Of particular importance is the emphasis on the selection of patients for minimally invasive procedures, aimed at determining the proper role for these tools in respiratory medicine.

A particular quality of the book is that the role of surgery in malignant diseases, like lung cancer or mesothelioma, is fairly presented, based on the evidence, but without useless literature data and without highlighting data that favour surgery as preferable option.

Fifteen appendices at the end of the book contain illustrations and schemes clarifying standard radiography, thoracic $\mathrm{CT}$, spirometry and bronchoscopy, novelties in the TNM (tumour, node, metastasis) classification of lung cancer and oesophageal carcinoma, mesothelioma staging system and pleural effusion diagnostic algorithm.

The authors should be congratulated because they have succeeded in being absolutely up to date, but also because the reader simply feels that they have major personal experience in all the topics covered. This book will "survive" the concurrence of many online learning resources, which are very useful in respiratory medicine in general, especially in surgical disciplines.

In brief, in the era of rapidly accumulating knowledge, together with the decreasing invasiveness of surgery and increasing invasiveness of nonsurgical procedures, this book will help to define the appropriate balance between them in order to improve overall treatment outcomes. 